\title{
IECA E BRA: EXISTEM DIFERENÇAS?
}

\author{
ACE AND ARBS : ARE THERE DIFFERENCES?
}

Erika M. G. Campana, ${ }^{1}$ Andréa Araujo Brandão'

\section{RESUMO}

O bloqueio do sistema renina angiotensina aldosterona é uma estratégia fundamental no tratamento e prevenção da doença cardiovascular. No contexto da hipertensão arterial (HAS), os inibidores da enzima conversora de angiotensina e os bloqueadores dos receptores de angiotensina compõem, juntamente com os diuréticos tiazídicos e os antagonistas dos canais de cálcio; o tripé fundamental no tratamento farmacológico da HAS. Adicionalmente, estas classes de fármacos são comumente usadas em pacientes com insuficiência cardíaca, doença arterial coronariana, diabetes e doença renal crônica. Neste ponto de vista os autores discutem as semelhanças e diferenças entre inibidores da enzima conversora de angiotensina e os bloqueadores dos receptores de angiotensina, demonstrando que, à luz dos conhecimentos disponíveis atualmente na literatura, é possível reconhecer que essas duas classes de fármacos são diferentes e têm efeitos clínicos distintos.

Descritores: Hipertensão; Tratamento; Sistema Renina Angiotensina Aldosterona; Proteção.

\section{ABSTRACT}

Blockade of the renin angiotensin aldosterone system is a fundamental strategy in the treatment and prevention of cardiovascular disease. In the context of arterial hypertension, angiotensin-converting enzyme inhibitors and angiotensin receptor blockers make up, along with thiazide diuretics and calcium channel antagonists; the fundamental tripod in the pharmacological treatment of SAH. In addition, these classes of drugs are commonly used in patients with heart failure, coronary artery disease, diabetes and chronic kidney disease. From this point of view, the authors discuss the similarities and differences between angiotensinconverting enzyme inhibitors and angiotensin receptor blockers, demonstrating that in light of the knowledge currently available in the literature, it is possible to recognize that these two classes of drugs are different and have distinct clinical effects.

Descriptors: Hypertension; Treatment; Renin Angiotensin Aldosterone System;Protection.

O bloqueio do sistema renina angiotensina aldosterona (SRAA) é uma estratégia fundamental no tratamento e prevenção da doença cardiovascular. No contexto da hipertensão arterial (HAS), os inibidores da enzima conversora de angiotensina (IECA) e os bloqueadores dos receptores de angiotensina (BRA) compõem, juntamente com os diuréticos tiazídicos e os antagonistas dos canais de cálcio; o tripé fundamental no tratamento farmacológico da HAS. Adicionalmente, estas classes de fármacos são comumente usados em pacientes com insuficiência cardíaca, doença arterial coronariana, diabetes e doença renal crônica. ${ }^{1}$

Ainda que atuem, promovendo o bloqueio do SRAA, IECA e BRA possuem mecanismos de ação distintos. Os IECA inibem a formação de angiotensina II e consequentemente seus efeitos através do receptor de angiotensina tipo 1 (AT1) (vasoconstrição, crescimento celular, retenção de sódio e água, ativação simpática) e do receptor da angiotensina II tipo 2 (AT2). Além disso, inibem a degradação da bradicinina. Já os BRA promovem inibição seletiva de receptores AT1 da angiotensina II, resultando em vasodilatação incremental, atividade antiproliferativa e natriurese. ${ }^{1}$

A primeira importante diferença entre IECA e BRA relaciona-se aos efeitos benéficos da inibição da degradação da bradicinina, com consequente aumento dos seus níveis circulantes promovidos pelos IECAs. Níveis aumentados de bradicinina liberam óxido nítrico e aumentam a síntese de prostaglandinas vasoativas, contribuindo para efeito vasodilatador adicional. Esta cascata autócrina associada com bradicinina, óxido nítrico e prostaglandinas vasoativas não é observada com o uso dos BRA..$^{1-3}$ Entretanto, vale destacar que a bradicinina tem sido implicada na patogênese da tosse associada ao uso de IECA. Portanto, utilizando como parâmetro de comparação o mecanismo de ação e suas implicações, já se pode observar que IECA e BRA não são iguais.

O segundo ponto de comparação relevante envolve os estudos clínicos com ambos os fármacos. A metanálise de van Vark et al. ${ }^{4}$ avaliou o risco de mortalidade total com o uso de IECA e BRA. Os autores avaliaram 20 ensaios clínicos e o

1. Universidade do Estado do Rio de Janeiro (UERJ), Rio de Janeiro, RJ, Brasil.

Correspondência: Erika Campana. Rua São Januário, no159/11O3 B. Fonseca - Niteroi/RJ - CEP: 24130-386. unigpesqmed@gmail.com

http://dx.doi.org/10.47870/1519-7522/2020270392-93 
impacto sobre os desfechos cardiovasculares (CV), mortalidade total e CV, envolvendo 158.998 pacientes (71.401 usando bloqueadores do SRAA; e 87.597 controles). O bloqueio do SRAA foi associado a uma redução significativa de 5\% na mortalidade por todas as causas (HR: 0,95; IC 95\%: 0,91-1,00; $p=0,032)$ e uma redução de $7 \%$ na mortalidade cardiovascular (HR: 0,93; IC 95\%: 0,88-0,99; p=0,018). A avaliação apenas dos estudos que utilizaram os IECA demonstrou uma redução significativa de $10 \%$ no risco de mortalidade por todas as causas (HR: 0,90; IC 95\%: 0,84-0,9; p=0,004), enquanto nenhuma redução na mortalidade total foi demonstrada com os BRA (HR: 0,99; IC 95\%: 0,94-1,04; p=0,683).

Duas metanálises recentes ${ }^{5,6}$ estudaram o papel dos bloqueadores do SRAA em pacientes pós-infarto agudo do miocárdio. Na primeira (R6), ${ }^{5}$ os autores avaliaram a mortalidade cardiovascular e por todas as causas aos 12 meses após o infarto agudo do miocárdio, em pacientes que receberam IECA ou BRA na admissão hospitalar. Foram analisados 12.481 pacientes do Registro de Infarto Agudo do Miocárdio da Coreia - Institutos Nacionais de Saúde. Na comparação direta, o tratamento com IECA foi associado a menor razão de risco para mortalidade cardiovascular (HR: 0,562; IC 95\% 0,4200,753) e total (HR: 0,567; IC 95\% 0,451-0,713).

Na segunda metanálise, ${ }^{6} 13.873$ pacientes com infarto agudo do miocárdio e submetidos à angioplastia primária com sucesso foram divididos em dois grupos de tratamento: betabloqueador em combinação com IECA ( $n=10.393)$ e betabloqueador em combinação com BRA ( $n=3.480$ ); o período de acompanhamento foi de 2 anos. A incidência de eventos cardiovasculares maiores (HR: 1,210; IC 95\% 1,010- 1,451; $p=0,039)$ no grupo betabloqueador com BRA foi significativamente maior que no grupo betabloqueador com IECA após angioplastia primária. Os resultados das duas metanálises reforçam as diferenças entre IECA e BRA em relação a efeitos clínicos.

Brugts JJ et al.7 realizaram metanálise envolvendo 18 estudos prospectivos, randomizados e controlados, para avaliação da morbidade e mortalidade (68.343 participantes usando um bloqueador do SRAA; 84.543 participantes controle). A metanálise avaliou os NNT para mortalidade por todas as causas, mortalidade cardiovascular, infarto do miocárdio e acidente vascular cerebral. O NNT correspondente para evitar um evento de morte por todas as causas foi $67 \mathrm{com}$ IECA versus 335 com BRA, uma morte cardiovascular: 116 com IECA versus 409 com BRA, e infarto agudo do miocárdio: 80 com IECA versus 338 com BRA, todos com diferença estatisticamente significativa. O NNT para evitar um evento de acidente vascular cerebral, foi mais favorável aos BRA (337 com IECA versus 131 com BRA), embora essa diferença não tenha sido estatisticamente significativa.

Estudos em contextos clínicos específicos também mostraram o benefício clínico de proteção cardiovascular com o uso de IECA, tais como: pós infarto agudo do miocárdio, insuficiência cardíaca, prevenção secundária do acidente vascular cerebral, e doença renal crônica com proteinúria. Assim, várias diretrizes internacionais recomendam o uso dos IECA como terapia preferencial, sendo os BRA considerados como terapia alternativa para pacientes intolerantes aos IECA. ${ }^{1,8-11}$ À luz dos conhecimentos disponíveis atualmente na literatura, é possível reconhecer que IECAs e BRAs são classes de fármacos diferentes com efeitos clínicos distintos.

\section{REFERÊNCIAS}

1. Malachias MVB, Plavnik FL, Machado CA, Malta D, Scala LCN, Fuchs S; Sociedade Brasileira de Cardiologia. 7a Diretriz Brasileira de Hipertensão Arterial. Arq Bras Cardiol. 2016; 107(3): 1-83.

2. Messerli FH, Bangalore S, Bavishi C, Rimoldi SF. Angiotensin-Converting Enzyme Inhibitors in Hypertension. To Use or Not to Use? J Am Coll Cardiol. $2018 ; 71: 1474-82$.

3. Taddei S, Bortolotto L. Unraveling the Pivotal Role of Bradykinin in ACE Inhibitor Activity. Am J Cardiovasc Drugs. 2016;16(5):309-21.

4. van Vark LC, Bertrand M, Akkerhuis KM, Brugts JJ, Fox K, Mourad JJ, et al. Angiotensin-converting enzyme inhibitors reduce mortality in hypertension: a meta-analysis of randomized clinical trials of renin-angiotensin-aldosterone system inhibitors involving 158,998 patients. Eur HeartJ. 2012; 33(16):2088-97.

5. Choi IS, Park IB, Lee K, Ahn TH, Kim JH, Ahn Y, et al; Korea Acute Myocardial Infarction Registry-National Institutes of Health (KAMIR-NIH) investigators. Angiotensin-Converting Enzyme Inhibitors Provide Better Long-Term Survival Benefits to Patients With AMI Than Angiotensin II Receptor Blockers After Survival Hospital Discharge. J Cardiovasc Pharmacol Ther. 2018; 21:1074248418795897.

6. Kim YH, Her AY, Jeong MH, Kim BK, Lee SY, Hong SJ, et al. Comparison Between Beta-Blockers with Angiotensin-Converting Enzyme Inhibitors and Beta-Blockers with Angiotensin II Type I Receptor Blockers in STSegment Elevation Myocardial Infarction After Successful Percutaneous

Coronary Intervention with Drug-Eluting Stents. Cardiovasc Drugs Ther 2019;33(1):55-67.

7. Brugts JJ, van Vark L, Akkerhuis M, Bertrand M, Fox K, Mourad JJ, et al. Impact of renin-angiotensin system inhibitors on mortality and major cardiovascular endpoints in hypertension: A number-needed-to-treat analysis. Int J Cardiol. 2015;(15)181:425-9.

8. Task Force of the Latin American Society of Hypertension. Guidelines on the management of arterial hypertension and related comorbidities in Latin America. J Hypertens. 2017;35(8):1529-45.

9. Nerenberg KA, Zarnke KB, Leung AA, Dasgupta K, Butalia S, McBrien K, et al. Hypertension Canada's 2018 Guidelines for Diagnosis, Risk Assessment, Prevention, and Treatment of Hypertension in Adults and Children. Can J Cardiol. 2018;34(5):506-525.

10. Whelton PK, Carey RM, Aronow WS, Casey DE Jr, Collins KJ, Dennison Himmelfarb C et al. 2017 ACC/AHA/AAPA/ABC/ACPM/AGS/APhA/ASH/ ASPC/NMA/PCNA Guideline for the Prevention, Detection, Evaluation, and Management of High Blood Pressure in Adults: Executive Summary: A Report of the American College of Cardiology/American Heart Association Task Force on Clinical Practice Guidelines. Hypertension. 2018;71(6):1269-1324.

11. Williams B, Mancia G, Spiering W, Rosei EA, Azizi M, Burnier M, et al. 2018 ESH/ESC Guidelines for the management of arterial hypertension. Eur Heart J. 2018;39(33):3021-04 